\title{
Aiming for G7 Master Compliance through a Color Managed Digital Printing Workflow (CMDPW): Comparison of Compliance with Output Device Profile (ODP) vs. Device Link Profile (DLP)
}

\section{ABSTRACT}

The purpose of this applied research was to determine the influence of device link profile (DLP) in the color reproduction aimed at the G7 master compliance. The quality of digital color printing is determined by these influential factors: screening method applied, type of printing process, ink (dry-toner or liquid-toner), printer resolution and the substrate (paper). For this research, only the color printing attributes such as the $G 7$ colors hue and chroma, gray balance, and overall color deviations were analyzed to examine the significant differences that exist between the two output profiles [Output Device Profile (ODP) vs Device Link Profile (DLP)]. These are the color attributes which are monitored and managed for quality accuracy during the printing. Printed colorimetry of each profile from the experiment was compared against G7 ColorSpace GRACoL 2013 (CGATS21-2-CRPC6) in CIE L* $a^{*} b^{*}$ space using an IDEAlliance (Chromix/Hutch Color) Curve 4.2.4 application interface with an X-Rite spectrophotometer with an i1iO table. The measured data of each profile were run through this application (Curve 4.2.4). The data were analyzed by using the Verify Tool of the Curve 4.2.4 application to determine the pass/fail of G7 master compliance levels using G7 ColorSpace tolerances (G7 Grayscale, G7 Targeted, and G7 Colorspace). Analyzed data from the experiment revealed that the printed colorimetric values of each profile (G7 Grayscale, G7 Targeted, and G7 Colorspace) are in match (aligned) with the $\mathrm{G} 7$ master compliance levels (reference) target) colorimetric values (G7 Grayscale, G7 Targeted, and G7 Colorspace). Therefore, the press run was passed by the Curve 4 application for both the profiles used/tested towards aiming for G7 master compliance.
Haji Naik Dharavath

Central Connecticut State University, Department of Computer Electronics \& Graphics Technology, New Britain, USA

Corresponding author: Haji Naik Dharavath e-mail:dharavathh@ccsu.edu

First received: 12.2 .2020 Accepted: 16.11.2020.

\section{KEY WORDS}

G7, Calibration, Color, Device Link, Output Profile, Colorimetry, Gamut, Screening

\section{Introduction}

G7 stands for grayscale (or gray) plus the seven primary and secondary colors known as the subtractive and additive: Cyan, Magenta, Yellow, Black (CMYK) and Red, Green, Blue (RGB). G7 is a method which specifies cali- bration procedures for printing visually acceptable colors with an emphasis on matching colorimetrically derived aim-points for the print reproduction processes to print with a common visual appearance. Today, this method (G7) is used in many applications of printing such as offset lithography, flexography, and digital (color laser 
or inkjet). It uses a pre-defined one-dimensional neutral print density curve (NPDC) to match neutral tonality/gray balance. G7 specifications are owned by International Digital Enterprise Alliance (International Digital Enterprise Alliance- IDEAlliance, 2014) and the colorimetric formulas of the G7 are defined in the American National Standards Institute and the Committee on Graphic Arts Technology Standards/Technical Report (ANSI/CGATS TR015). Published reports reveal there are three ways G7 master compliance can be achieved: a) output device NPDC to G7 NPDC [P2P251x target image], b) use of output device ICC profile, and c) the use of device link profile (DLP = source as GRACoL2013 ICC profile + the destination device ICC profile). G7 master compliance includes three levels in the $\mathrm{G} 7$ master qualification: $\mathrm{G} 7$ Grayscale, G7 Targeted, and G7 Colorspace. These levels demonstrate $\mathrm{G} 7$ master capabilities of a print facility.

\section{Review of literature}

Digital color print reproduction involves physical/ mechanical interaction among the imaging cylinder, dry/ liquid toner, and the substrate (Avramovic \& Novakovic, 2012). The outcome of this interaction is the color print. Color can be viewed as a science where the optical aspects of color are quantitatively analyzable and measurable. The human eye, however, perceives color more subjectively, which poses a challenge at times for the printing and image reproduction industry. The quality of a color image reproduced through any printing process (digital or traditional) is largely influenced by the properties of paper. While paper is considered a commodity, its properties are a long way from being standardized (Wales, 2009). Additional attributes must be monitored in order to produce quality printed materials; a high-quality color image. The press operator must carefully manage several print parameters, such as the source colors (a source profile of ISO or ANSI standard), press calibration, press characterization (device destination profile), and the screening option. Without controlling these parameters to a print job, a color mismatch would result. A modern and up-to-date commercial printing workflow requires a Color Management System (CMS) to produce a quality color printing. A CMS enables the color producer (printer operator or the designer) to deliver accurate output colors regardless of device color capacities with the use of proper color management techniques (see Figure 1). Analyzing the color image by examining its quantitative attributes eliminates the subjective judgment of color quality evaluation of printed colors or colors in nature. Advancements in science and engineering, however, have allowed print and graphic professionals to apply scientific research methods across printing workflow. Applying these methods heightens the importance of proper print production workflow. Gray balance represents the combination of specific amounts of cyan, magenta, and yellow inks to produce a neutral shade of gray. With slight increases in cyan pigment required to produce a neutral gray, shifts in hue will occur with any imbalance of these three components. In addition to the color gamut, the gray balance is an additional requirement for pleasing color-reproduction. In large part, the imbalance is due to impurities of the inks, chromaticity deviation of the substrates, or other attributes. To establish the proper gray balance for a specific process, a full set of tint charts can be reproduced. Careful evaluation of the printed tint charts will provide the specific values for that specific reproduction process. The ISO 12647-7 document states that the gray balance can be printed and measured at the CMY overlap (overlap of $\mathrm{C}=50 \%$, $M=40 \%$, and $Y=40 \%$ ). The deviation can be determined from the calculation of $\Delta \mathrm{H}^{*}$ (deviation of hue, $\mathrm{h}^{*}$ ) or $\Delta \mathrm{C}^{*}$ (deviation of chroma, $\mathrm{c}^{*}$ ) and it requires the colorimetric data of CMY overlap printing from the $L^{*} a^{*} b^{*}$ model.

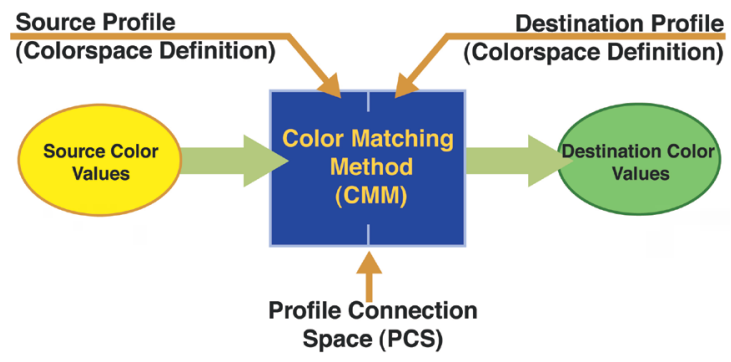

» Figure 1: Schematic of PCS of CMS (Courtesy of Adobe Systems, Inc.)

\section{G7 Grayscale}

This is the fundamental level of G7 commonly seen in most color print reproduction. Regardless of printing process, if a digital printer or printing press reproduces the defined neutral tone ramp as a neutral gray, then all other colors in the reproduction are believed to be without colorcast. This is determined by printing a target specified on a stable printing system and then measuring the target using the correct ink/toner curves to bring the printing system into alignment with the $\mathrm{G} 7$ ideal neutral density curve. Aligning the various reproduction processes and obtaining the same neutral aim points is critical for consistent reproduction.

\section{G7 Targeted}

The secondary level of G7 is achieved when G7 grayscale is matched, and the solid ink measurements for primary, and secondary (CMY and RGB) are also within the G7 target specifications. This can be achieved through the absolute white point or using the substrate-relative conditions. However, G7 Targeted compliance is not limited to the reference print conditions in ISO 12647-2 or in ISO/ PAS 15339. The G7-calibrated dataset can be used as a G7 reference print condition. G7 Targeted achievement cer- 
tifies that the facility not only conforms to G7 Grayscale, but it can also achieve a higher level of compliance.

\section{G7 Colorspace}

The highest level of G7 compliance, and the most stringent is the $\mathrm{G} 7$ Colorspace. It includes all the requirements of the G7 Targeted level and, therefore, the G7 Grayscale level. This also includes the matching of an entire Reference Print Condition (RPC). This level of control demonstrates that the reproduction maintains an extremely tight tolerance throughout the complete color space. An entire TC1617x target is printed and compared against the specific color space with all 1617 patches held to within a tight tolerance. This assures the printing system will reproduce the entire color space, not just the primary and secondary colors of CMYK and RGB. The G7 Colorspace can also relate to either the absolute white point or the substrate-relative aim values.

\section{Purpose of the research}

The purpose of this applied research was to demonstrate the use of a complete color managed workflow (CMW) and meet the specified G7 master compliance levels by creating and using output device ICC profiles. The experiment was conducted in a color managed digital printing workflow (CMDPW) to determine the effect that ODP and DLP have on the G7 master compliance: Comparison of Compliance with Output Device Profile (ODP) vs. Device Link Profile (DLP) of Multicolor Digital Printing. It was aimed at achieving the G7 master compliance through an ICC based CMW. As stated earlier, the $\mathrm{G} 7$ master compliance print evaluation can be achieved by use of the output device ICC profile (ODP or DLP) for printing. This experiment adopts both methods to achieve the compliance and compare with each other. G7 master compliance includes three compliance levels in the $\mathrm{G} 7$ master qualification: G7 Grayscale, G7 Targeted, and G7 Colorspace. These levels demonstrate $\mathrm{G} 7$ master capabilities of a print facility. The G7 calibration method, using the P2P251x target, was NOT considered to derive the device NPDC to compare with G7 NPDC for print (or press) runs 1, 2, 3, etc.

\section{Limitations of the Research}

For this research, limitations in the technology of the graphics laboratory were acknowledged. Prior to printing and measuring the samples, the digital color output printing device, and color measuring instruments (spectrophotometer and densitometer) were calibrated against the recommended reference. The print conditions associated with this experiment were characterized by, but not restricted to, the inherent limitations: colored images (TC1617x, ISO300, and ISO12647-7) chosen for printing. Additionally, the desired rendering intent applied, type of digital printer, type of paper, type of toner, resolution, screening technique, color output profiles, and calibration data applied are acknowledged. Several variables affected the facsimile reproduction of color images in the CMDPW, and most were mutually dependent. The scope of the research was limited to the color laser (electrophotographic) digital printing system (printing proof/printing), substrates, types of color measuring devices, color management and control applications (data collection, data analysis, profile creation, and profile inspection) used within the university graphics laboratory. Findings were not expected to be generalizable to other CMDPW environments. It is quite likely, however, that others will find the method used and data collected both useful and meaningful. The research methodology, experimental design, and statistical analysis were selected to align with the purpose of the research, taking into account the aforementioned limitations.

\section{Research methodology}

The digital color printing device used in this experiment is a Konica-Minolta bizHub C6000 Digital Color Press. It uses a Creo IC-307 raster image process (RIP) application (front-end system). A two-page custom test image $\left(12^{\prime \prime} \times 18^{\prime \prime}\right.$ size) was created for proofing and printing use for the experiment (See Figures 2 \& 2A). The test target contained the following elements: an ISO 300 and generic images for subjective evaluation of color, an ISO 12647-7 Control Strip (2013, three-tier), and a TC1617x target for gamut/profile creation. Glass, G.V. \& Hopkins, K. D. (1996) provides an objective method to determine the sample size when the size of the total population is known. The following formula was used to determine the required sample size, which was 80 (n) printed sheets for each group used in this study:

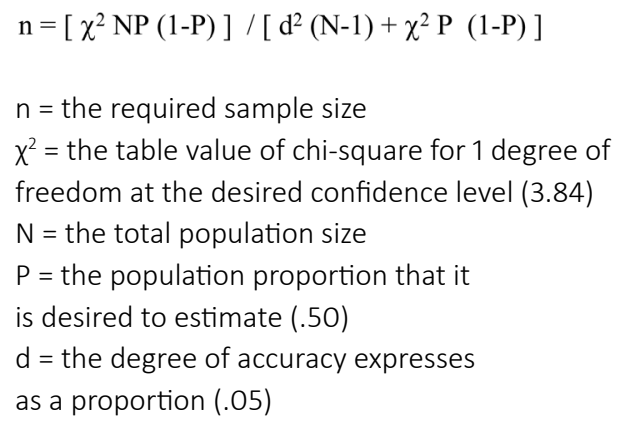

Table 1 presents the variables, materials, conditions, and equipment associated with this experiment. Colorimetric, Densitometric, and Spectrophotometric data were extracted by using an X-Rite Eye-One Spectrophotometer and an X-Rite i1iO Scanning Spectrophotometer from the color printed samples for the analysis. For both profiles [ODP and DLP (groups, $K=2$ )], a total of 200 samples of target color images were printed, 100 prints with each profile noted by letter " $N$ " $(N=100)$. 
Table 1

Experimental and Controlled Variables

\begin{tabular}{|c|c|}
\hline Variable & Material/Condition/Equipment \\
\hline Test image & Custom Test Target, 2 pages \\
\hline Control strips/targets & ISO 12647-7 (2013), TC1617x \\
\hline Other Images & B/W and Color for Subjective Evaluation \\
\hline Profiling Software & X-Rite i1PROFILER 1.8 \\
\hline Profile Inspection Software & Chromix ColorThink-Pro 3.0 \\
\hline Image Editing Software & Adobe PhotoShop-CC \\
\hline Page Layout Software & Adobe InDesign-CC \\
\hline Source Profile (RGB) & Adobe 1998.icc \\
\hline Destination Profile (CMYK) & Custom, Konica-Minolta.icc \\
\hline Reference/Source Profile (CMYK) & GRACoL2013.icc \\
\hline Color Management Module (CMM) & Adobe (ACE) CMM \\
\hline Rendering Intents & Absolute \\
\hline Computer \& Monitor & Dell OPTIPLEX/LCD \\
\hline Raster Image Processor (RIP) & Creo IC-307 Print Controller \\
\hline Printer & Konica-Minolta bizHub C6000 Color Laser \\
\hline Achieved CMYK SID for all print runs (AM Screen) & $C=1.45 ; M=1.36 ; Y=0.90 ;$ and $K=1.73$ \\
\hline Screens and Screen Ruling & AM and 190 LPI \\
\hline Print Resolution & $600 \times 600 \mathrm{DPI}$ \\
\hline Toner & Konica-Minolta Color Laser \\
\hline Type of Paper Weight/thickness & Hammermill 100LB Matte Coated, Sheetfed \\
\hline Type of Illumination/Viewing Condition & D50 \\
\hline Color Measurement Device(s) & $\begin{array}{c}\text { X-Rite Eye-One PRO Spectrophotometer with Status T, } \\
20 \text { angle, and i1iO Scanning Spectrophotometer }\end{array}$ \\
\hline Data Collection/Analysis Software & IDEAlliance/Chromix Curve 4.0 / MS-Excel \\
\hline
\end{tabular}

Of 100 samples of each group, 80 samples $(n=80)$ were randomly selected and measured, noted by the letter " $n$ " $(n=80)$. This sample size is needed to make sure the reliability of data is accurate. It is well documented that a large sample size is more representative of the sampling population (subjects).

\section{G7 Compliance for Digital Color Press (printer)}

Prior to printing the patches/target image, the printer was calibrated for amplitude modulated (AM) screening according to its manufacturer specifications. A calibration process means standardizing the performance of the devices according to the device manufacturer specifications so that the results of the devices are repeatable. The calibration curve consists of the maximum printable densities of each color (CMYK) on the press (See Figure 3). Test target TC1617x was used for the output device profile creation process.

In a generic color managed digital printing workflow, digital front-end (DFE) platforms (raster image processor or RIP) of digital printers (or presses) offer opportunities for the user (or press operator) to manipulate the output color quality to meet the expected demand of the customer. The AM screening option offered the ability to set printer resolution at $600 \mathrm{DPI}, 1200 \mathrm{DPI}$, and 2400 DPI. But as a choice only the 600 DPI resolution was selected for both profiles to keep the print parameters/ variables consistent throughout the experiment.

\section{Output Device Profile (ODP) for G7 Compliance}

The target image (TC1617x) was placed into an Adobe InDesign-CC layout of 12" W x 18" H size and a .PDF file was created without any image/color compression technique (see Figures $2 \& 2 A$ ). Hammermill brand, 100 LB matte-coated digital color printing paper $12^{\prime \prime} \times 18^{\prime \prime}$ was used for printing the target image in the experiment. Prior to printing the TC1617x target (See Figure 2A) for creating the device profile, the printer was calibrated. The calibration data (range of CMYK densities) were saved in the calibration lookup tables of the raster image processor (RIP) and a calibration curve was created (See Figure 3). A total of 100 sheets/copies of TC1617x were printed with the calibration curve attached. Also, an amplitude modulated (AM) halftone screening technique with 190 lines per inch (LPI) and $600 \mathrm{DPI}$ as the printer resolution was applied during the printing of the target. No color management or color correction techniques were applied during the printing. Printed patches of TC1617x were measured in $\mathrm{CIE} \mathrm{L}^{*} a^{*} b^{*}$ space using the i1PROFILER application with an X-Rite spectrophotometer with an i1iO table and the data were run through this application. The printer profile (Output Device Profile) was created and stored at the right location on the computer. 
The profile format version is 4.00 and it is considered as the Output Device Profile (ODP) of AM screen-

ing. This profile was used as a destination profile
(DP) in the workflow. The source profile (SP) used in the experiment is a GRACoL2013 for characterized reference printing conditions-6 (CRPC-6).

\section{Aiming for G7 Master Compliance through a}

Color Managed Workflow: Comparison of Compliance with Output Device Profile (ODP) vs. Device Link Profile (DLP) of Multicolor Digital Printing
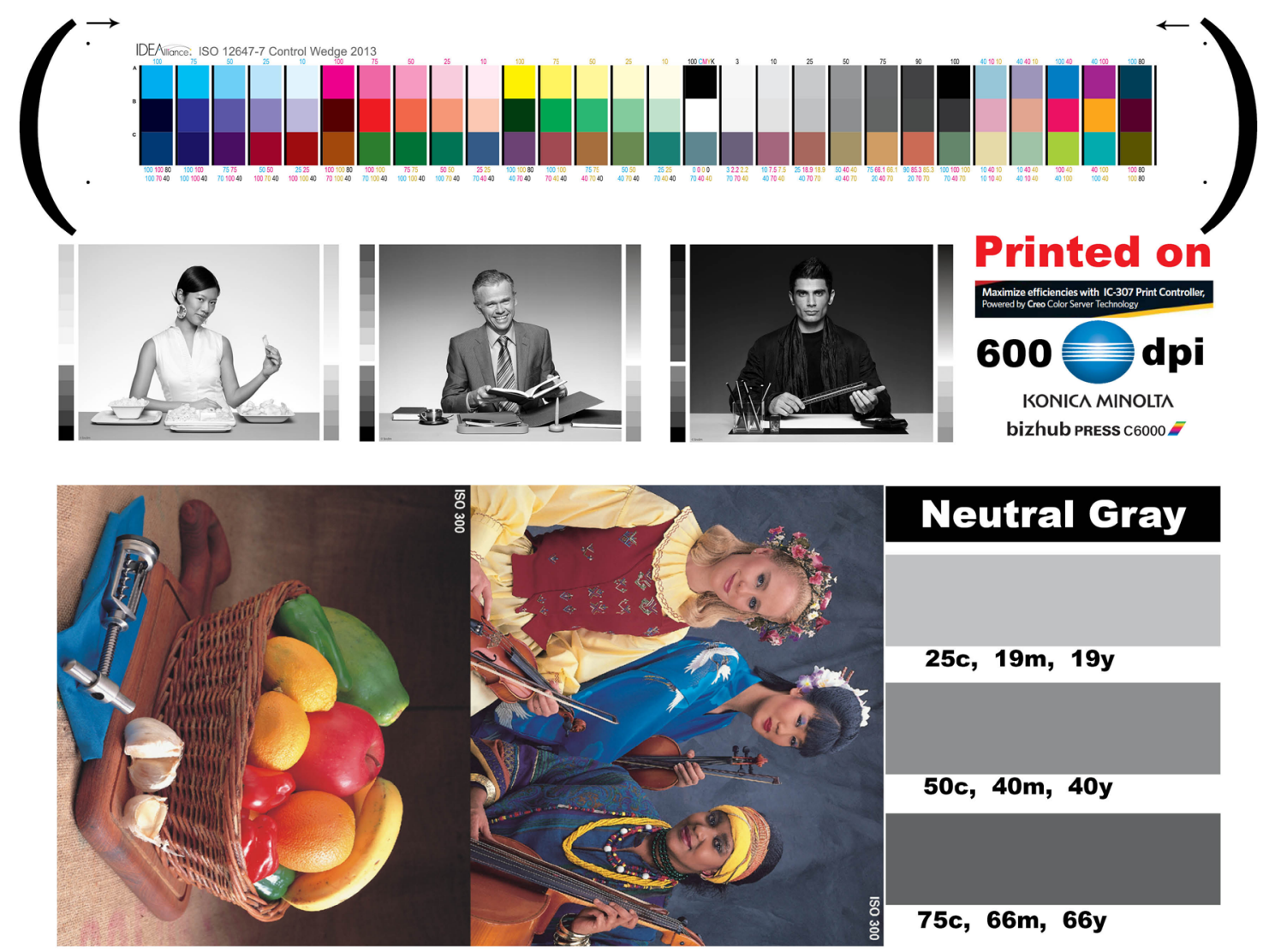

\section{Neutral Gray}
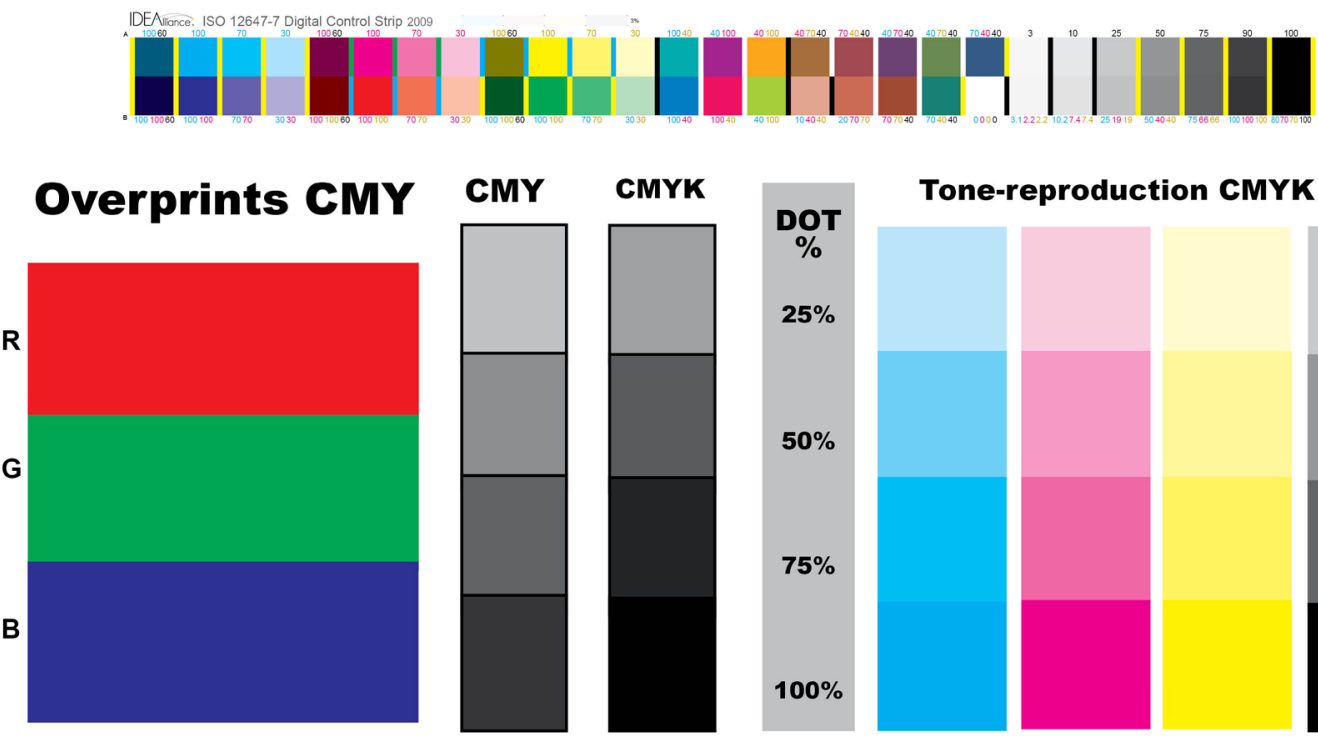

\section{Tone-reproduction CMYK scale}

» Figure 2: Test Image for the experiment (PAGE 01) 
Aiming for G7 Master Compliance through a Color Managed Workflow: Comparison of Compliance with Output Device Profile (ODP) vs. Device Link Profile (DLP) of Multicolor Digital Printing
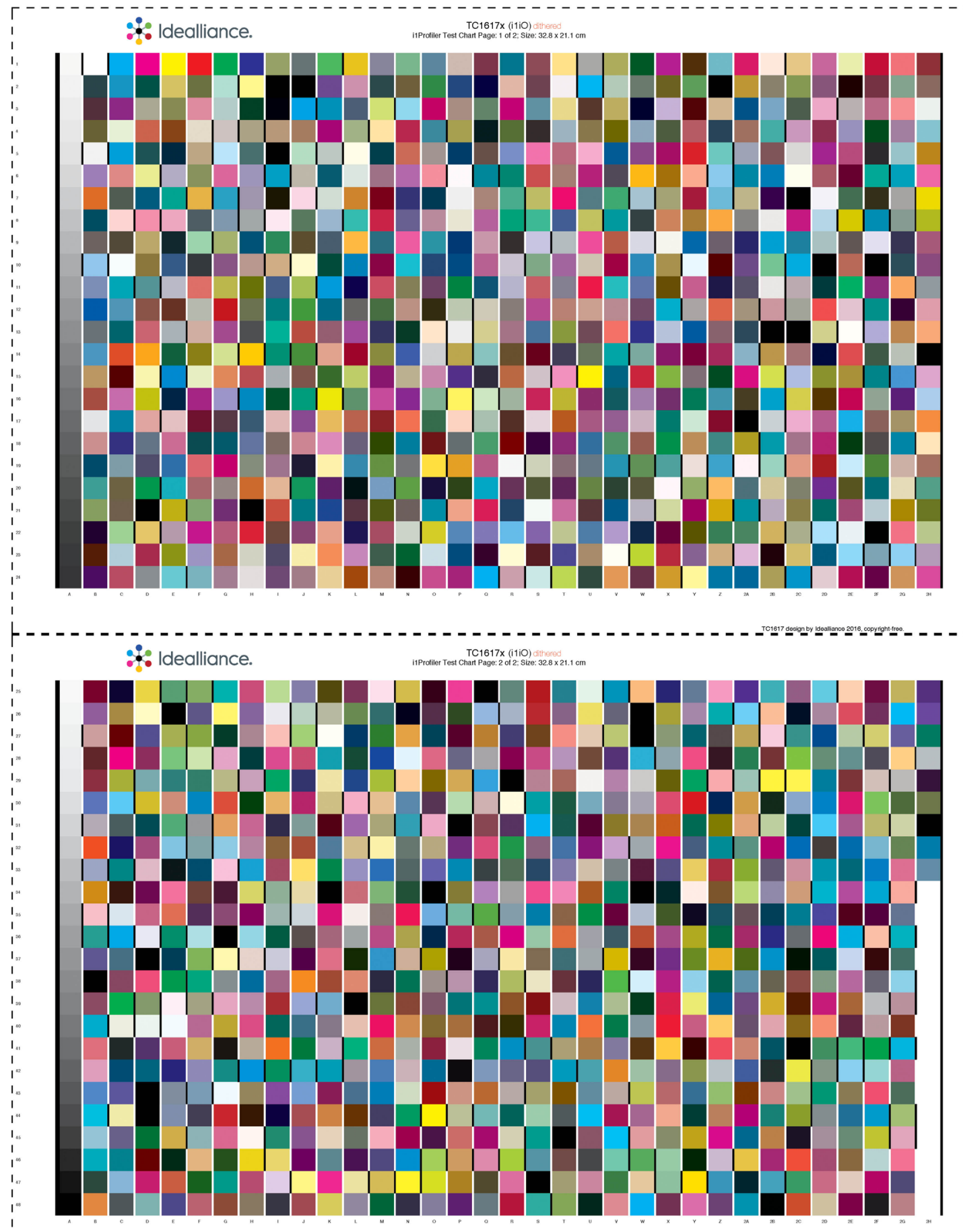

» Figure 2A: Test Image for the experiment (PAGE 02)

\section{Device Link Profile (DLP) for G7 Compliance}

In a generic color managed digital printing workflow, the color space of the input device (RGB) is transformed to the color space of the output device (CMYK) via the device independent $L^{*} a * b *$ color space or the profile connection space (PCS). This process (the DLP) requires two different profiles, namely a source (RGB) profile and a destination (CMYK) profile. There is no PCS in a color 
PRINTER CALIBRATION FOR G7 MASTER COMPLIANCE
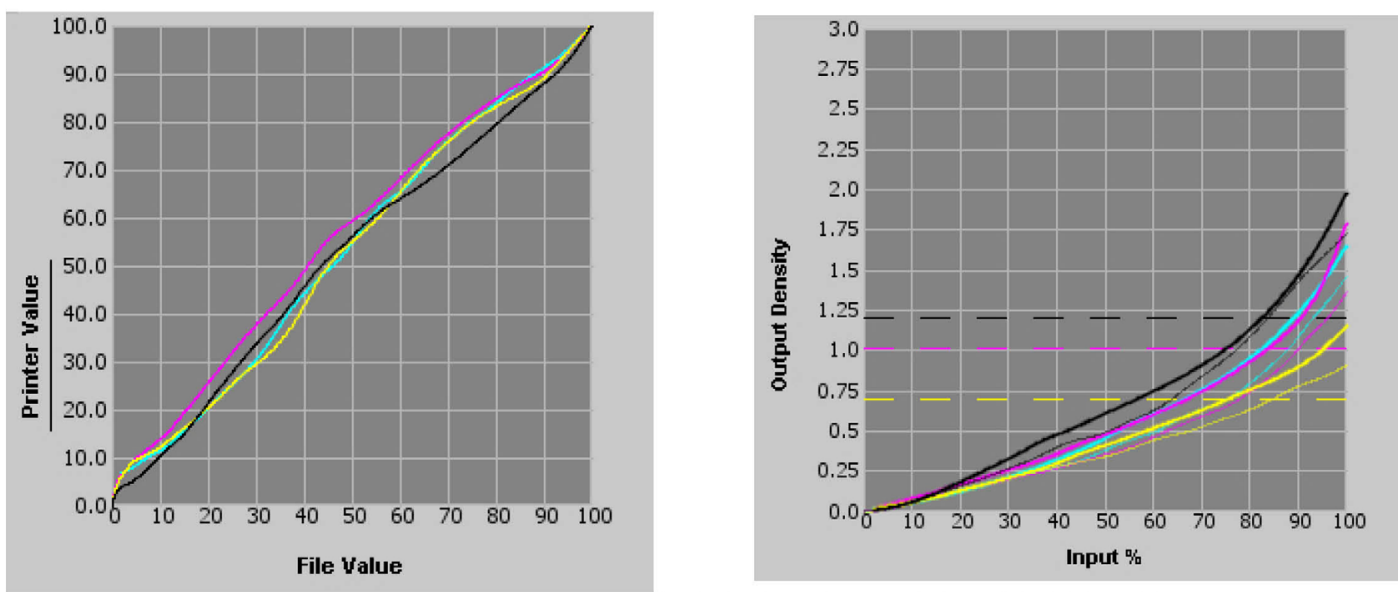

Measured Solid Ink Density (SID) of:

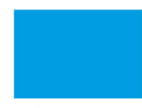

C

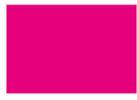

M

$1.45 \quad 1.36$

0.90

1.73

» Figure 3: Calibration of a Digital Press

managed digital printing workflow which uses a DLP. The source and the ODP are directly linked. Device link profiles are most commonly applied to direct CMYK-to-CMYK color transformations. An X-Rite i1PROFILER application was used to create the DLP by merging the source profile (GRACoL2013) and the ODP of the digital press. In this scenario the source profile used was GRACoL2013 for characterized reference printing conditions-6 (CRPC-6) and the destination device profile used was the ODP (Konica-Minolta $\mathrm{C} 6000$ bizHUB digital color press). So, the DLP used contained the two profiles.

\section{Printing for G7 Compliance}

Each profile (ODP vs DLP) used in the experiment was considered as a group, noted by letter " $K$ " $(K=2)$. A group involves a set of print parameters, such as: a digital halftone screening technique [amplitude modulate (AM)], the calibration curve (of AM screened), a color source profile [General Requirements for Applications in Commercial offset Lithography for characterized reference printing conditions-6 (GRACoL2013 for CRPC6)] or a DLP, and a color destination profile of a digital press (AM screened) or a DLP. As parameters illustrated in the figure 4 (Schematic Illustration of Sequence of Print Parameters for $G 7$ Compliance), test target of 12 " $\times 18$ " was printed for use in the experiment.

\section{Press Run 1: Printing with ODP}

A total of 100 sheets/samples were printed. The digital press calibration curve, AM screening destination profile, and the source profile (GRACoL 2013) all were applied during the printing. A total of 80 randomly pulled printed copies of TC1617x printed target images were measured against G7 ColorSpace GRACoL 2013 (CGATS21-2-CRPC6) in CIE L* $a^{*} b^{*}$ space using an IDEAlliance (Chromix/Hutch Color) Curve 4.2.4 application interface with an X-Rite spectrophotometer with an i1iO table. The measured data were combined/averaged to run through this application (Curve 4.2.4). The combined data set was analyzed by using the Verify Tool of the application to determine the pass/fail of G7 master compliance levels using G7 ColorSpace tolerances. Analyzed data from the experiment revealed that the printed colorimetric values (G7 Grayscale, G7 Targeted, and $\mathrm{G} 7$ Colorspace) are in match with the $\mathrm{G} 7$ master compliance levels (reference/target) colorimetric values (G7 Grayscale, G7 Targeted, and G7 Colorspace).

\section{Press Run 2: Printing with DLP}

A custom test target from the press run one was used for proofing and printing use for the experiment. A total of 100 sheets/samples were printed by enabling the color management technique for the use of DLP at the RIP. The digital press calibration curve and the DLP both were applied during the printing. A total of 80 randomly pulled printed copies of TC1617x printed target images were measured against G7 ColorSpace GRACoL 2013 (CGATS21-2-CRPC6) in CIE L* a* b* space using an IDEAlliance (Chromix/Hutch Color) Curve 4.2.4 application interface with an X-Rite spectrophotometer with an i1iO table and the data were combined to run through this application. Color measurement and analysis steps used in the printing with ODP process 
(previous section) were applied/followed for printing with DLP process. Printed colors with DLP were also found to be very accurate and the process was efficient.

\section{Data analysis \& research findings}

The colorimetric computation methods for G7 compliance were used to analyze the collected data and presented in the following pages/tables. Subjective judgment on color difference or any deviation was not used in this particular study because the subjective judgment of color difference could differ from person to person. For example, people see colors in an image not by isolating one or two colors at a time (Goodhard \& Wilhelm, 2003), but by mentally processing contextual relationships between colors where the changes in lightness (value), hue, and chroma (saturation) contribute independently to the visual detection of spatial patterns in the image (Goodhard \& Wilhelm, 2003). Instruments, such as colorimeters and spectrophotometers, eliminate subjective errors of color evaluation perceived by human beings. In comparing the color differences between two colors, a higher deviation ( $\Delta \mathrm{E}$ or $\Delta \mathrm{H}$ or the $\Delta \mathrm{C}$ ) is an indication that there is more color difference and a lesser deviation ( $\Delta \mathrm{E}$ or $\Delta \mathrm{H}$ or the $\Delta \mathrm{C}$ ) is an indication of less color difference. In this scenario of the color measuring/evaluation stage, a consistent and standardized light source (D50 or D65) and angle of viewing $\left(2^{\circ}\right.$ or $\left.10^{\circ}\right)$ are important.

\section{CIE L*a* b*, Delta L* Delta E and Delta Chroma $(\Delta \mathrm{L}, \Delta \mathrm{E}$ and $\Delta \mathrm{C})$}

Colorimetric values of printed colors against original colors and the deviations (Delta's) can be used to determine the visual variation in overall colors, hue, chroma, and lightness. The $a^{*}, b^{*}$ coordinates correspond approximately to the dimensions of redness - greenness and yellowness - blueness respectively in the CIE L* $\mathrm{a}^{*} \mathrm{~b}^{*}$ color space and are orthogonal to the $L^{*}$ dimension. Hence, a color value whose coordinates $a^{*}=b^{*}=0$ is considered achromatic regardless of its $L^{*}$ value. Calculation of $\Delta H^{*}$ requires colorimetric data from the $L^{*} a^{*} b^{*}$ model.
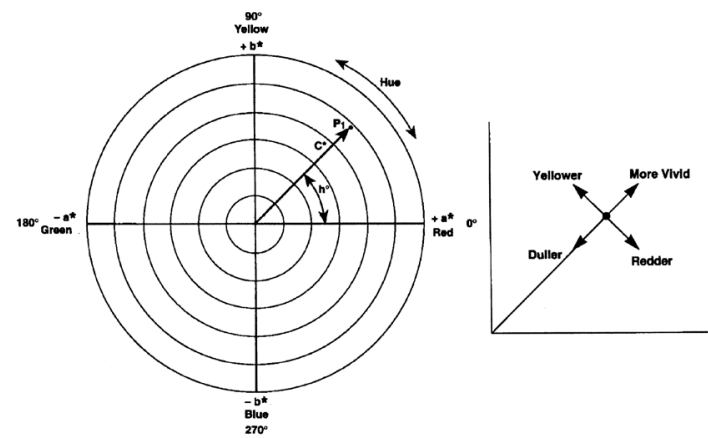

» Figure 5: Schematic of $L^{*} a^{*} b^{*} \& c^{*}, h^{*}$ Coordinates

Metric hue angle $h^{*}$ and $C^{*}$ are defined by the following formulas (Morovic, Green \& MacDonald, 2002).

AIMING FOR G7 MASTER COMPLIANCE THROUGH A COLOR MANAGED WORKFLOW SEQUENCE OF PRINT PARAMETERS FOR THE COMPLAINCE ODP VS. DLP

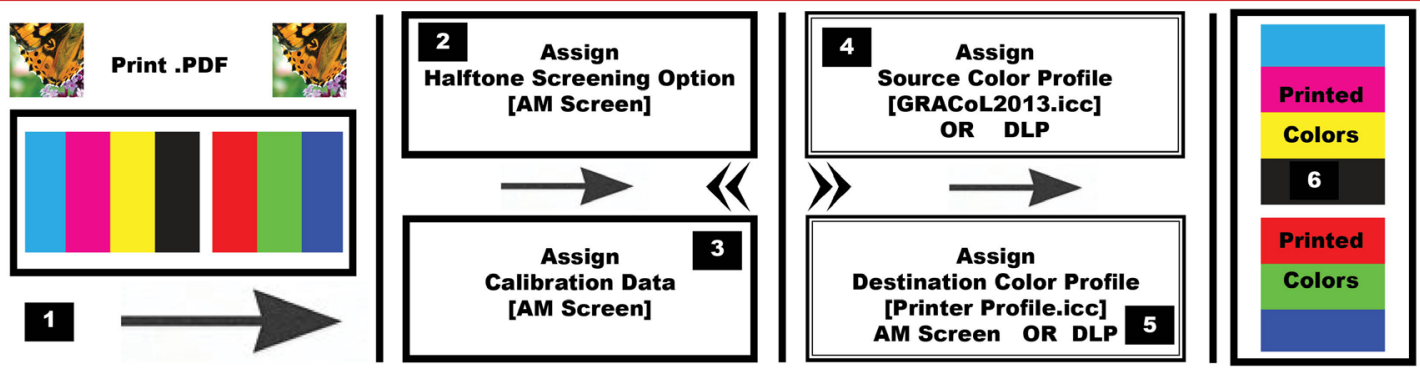

Color Output for G7 Compliance in a Color Managed Workflow (CMW): ODP vs DLP

\begin{tabular}{|c|c|c|c|c|c|}
\hline Group $=K$ & $\begin{array}{l}\text { Type of } \\
\text { Halftone Screen }\end{array}$ & $\begin{array}{l}\text { Destination } \\
\text { Profile }\end{array}$ & $\begin{array}{l}\text { Calibration } \\
\text { Curve }\end{array}$ & $\begin{array}{l}\text { Source } \\
\text { Profile }\end{array}$ & $\begin{array}{l}\text { Device Link } \\
\text { Profile (DLP) }\end{array}$ \\
\hline 1 [ODP] & $A M$ & $A M$ & $A M$ & GRACoL2013.icc & $\frac{\text { GRACoL2013.icc }}{+ \text { [plus] }}$ \\
\hline 2 [DLP] & N/A & N/A & AM & N/A & $\underline{O D P}$ \\
\hline
\end{tabular}

» Figure 4: Schematic Illustration of Sequence of Print Parameters for G7 Compliance 
Metric hue angle: $h *_{a b}=\tan ^{-1}\left(\frac{b *}{a *}\right)$

Where: $a^{*}, b^{*}$ are chromaticity coor-

dinates in $L^{*} a^{*} b^{*}$ color space

Chroma $\left(\mathrm{C}^{*}\right)=\left[\mathrm{a}^{2}+\mathrm{b}^{2}\right]^{1 / 2}$

Where: $a^{*}, b^{*}$ are chromaticity coordinates in $L^{*} a^{*} b^{*}$ color space

Calculation of $\Delta C^{*}$ (of two colors) and $\Delta \mathrm{L}^{*}$ requires colorimetric data from the $L^{*} a^{*} b^{*}$ model. Difference in the chroma $C^{*}$ of two colors (Reference vs. Printed) can be calculated by using the following formula (Green et al., 2002).

$\Delta$ Chroma $(\Delta \mathrm{C})=\mathrm{C}^{*}{ }_{1}-\mathrm{C}^{*}{ }_{2}$

Where: $1=\mathrm{C}^{*}$ of Reference Col-

or and $2=\mathrm{C}^{*}$ of Printed Color

Assessment of color is more than a numeric expression. It is an assessment of the difference in the color sensation (delta) from a known standard. In the CIELAB color model, two colors can be compared and differentiated. The expression for these color differences is expressed as $\triangle E$ (Delta $E$ or Difference in Color Sensation). The following equation is used to calculate the $\triangle E$ (Committee for Graphic Arts Technologies Standards - CGATS, 2003)

$$
\Delta E^{*}=\sqrt{\left(L_{1}-L_{2}\right)^{2}+\left(a_{1}-a_{2}\right)^{2}+\left(b_{1}-b_{2}\right)^{2}}
$$

Where: $1=$ Reference Color and $2=$ Printed Color

Chromaticness difference $(\Delta C h)$ is the difference between the reference chroma ( $a * 1$ and $b * 1)$ and the measured chroma ( $a * 2$ and $b * 2)$ of a gray balance control patch (C50, M40, Y40). Weighted Delta Chroma ( $w \Delta C h)$ is the delta $C h$ value after it is passed through a weighting curve that reduces the significance of Ch errors in the darker regions of the color. The weighting function is defined in the G7 specifications ([Technical Report (TR) 015] and the G7 master pass/fail document as follows (Chromix, Inc., 2019):

$$
\mathrm{w} \Delta \mathrm{Ch}=\Delta \mathrm{Ch} \times[1-\max (0,(\%-50) / 50 \times 0.75)]
$$

Delta $L^{*}\left(\Delta L^{*}\right)$ is the difference in the lightness between the reference and measured sample lightness regardless of any color. This makes $\Delta L^{*}$ the perfect metric for measuring tonality [Neutral Print Density Curve (NPDC)] error in G7. Colorimetrically, $\Delta L^{*}$ is the result of subtracting the $L^{*}$ of measured sample value from the reference $L^{*}$, as follows:

$$
\Delta \mathrm{L} *=\mathrm{L}^{*}-\mathrm{L}^{*}
$$

Where: $1=L^{*}$ of Reference Color and $2=L^{*}$ of Printed Color
Weighted Delta $L^{*}\left(w \Delta L^{*}\right)$ is the delta $L^{*}$ value after it is passed through a weighting curve that reduces the significance of $L^{*}$ errors in the darker regions of the color. The weighting function is identical to that for $w \Delta C h$, as follows (Chromix, Inc., 2019):

$\underline{\mathrm{w}} \Delta \mathrm{L}^{*}=\Delta \mathrm{L}^{*} \times[1-\max (0,(\%-50) / 50 \times 0.75)]$

\section{Overall Color Variation $(\triangle \mathrm{E})$ of ODP (TC1617x image) vs. GRACoL 2013 Ref.}

The CIE L* $a * b *$ values associated with the CMYK+RGB colors of printed image with ODP vs. G7 ColorSpace-GRACoL 2013 [CGATS21-2-CRPC6 (reference)] are compiled in Table 2. Numerical color differences $(\Delta E)$ were found when comparing the colors of printed image vs. G7 ColorSpace at all seven colors (CMYK+RGB). Also, noticeable visual color differences were found in the solid color area [lightness, color hue and chroma]. Overall, both groups of images have similar colors (see Figures $6,7,8$ and 9) with the exception of the printed image consisting of higher $L^{*}$ for red, magenta, and green, etc. This results in producing the higher $\Delta \mathrm{E}$ for these colors.

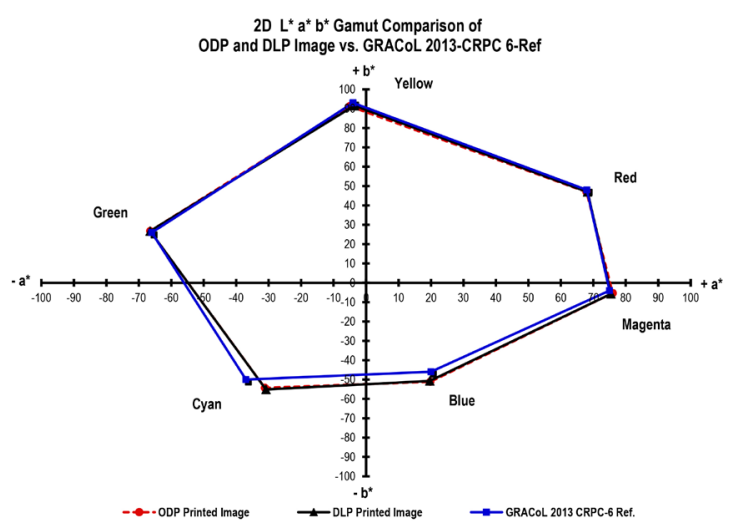

» Figure 6: Image (colors) printed with ODP and DLP vs. GRACoL 2013-CRPC-6 Ref.

This higher color deviation (red, magenta and green) could be the result of the substrate (paper) and toner used (age, condition, quality, etc.). These are the darker colors which produced lower L* value and in turn affected the higher deviation.

The 2D color gamut comparison (see Figures 6, 7, 8 and 9) reveals that the colors of the printed image closely match the reference colors. The goal was to determine the deviations among various attributes of color between these two groups of colors.

The comparison is an indication that, in a color managed workflow (CMW), color matching of a target image can be achieved from device to device regardless of device color characterization and original colors. Subjective judgment was not used for the color comparison. 
Table 2

Overall Color Variation of CMYK+RGB: Printed Image (TC1617x) vs. G7 ColorSpace

\begin{tabular}{|c|c|c|c|c|c|c|c|}
\hline \multirow{3}{*}{ Color(s) } & \multicolumn{3}{|c|}{ ODP Printed Image } & \multicolumn{3}{|c|}{ G7 ColorSpace / Target } & \multirow{3}{*}{$\begin{array}{c}\text { Color } \\
\text { Difference } \\
\text { 回E }\end{array}$} \\
\hline & $L^{*}$ & $a^{*}$ & $b^{*}$ & $\mathrm{~L}^{*}$ & $a^{*}$ & $b^{*}$ & \\
\hline & \multicolumn{3}{|c|}{$\begin{array}{l}\text { Color } 1 \\
\mathbf{N}=\mathbf{8 0}\end{array}$} & \multicolumn{3}{|c|}{$\begin{array}{l}\text { Color } 2 \\
\mathbf{N}=\mathbf{N} / \mathbf{A}\end{array}$} & \\
\hline White (W) & 97.22 & 2.79 & -9.45 & 97.22 & 2.79 & -9.45 & 0.00 \\
\hline Cyan & 57.22 & -31.17 & -54.27 & 57.40 & -36.88 & -55.84 & 2.06 \\
\hline Magenta & 51.19 & 75.97 & -5.30 & 49.22 & 77.93 & -7.09 & 2.13 \\
\hline Yellow & 90.86 & -5.06 & 91.29 & 91.10 & -2.43 & 92.93 & 1.47 \\
\hline Black (K) & 13.07 & 0.47 & 0.07 & 16.30 & 0.24 & -0.74 & 2.29 \\
\hline Red & 50.50 & 67.90 & 46.95 & 48.19 & 70.74 & 48.26 & 2.40 \\
\hline Green & 53.56 & -66.51 & 26.72 & 51.26 & -66.87 & 24.49 & 2.45 \\
\hline Blue & 27.06 & 19.75 & -51.14 & 25.62 & 21.19 & -50.24 & 1.71 \\
\hline TAC 300 & 24.27 & 0.01 & -1.95 & 23.56 & 0.45 & -1.34 & 1.01 \\
\hline TAC 400 & 9.91 & 0.72 & -1.04 & 8.99 & 0.17 & 0.61 & 1.90 \\
\hline
\end{tabular}

\section{Overall Color Variation $(\triangle E)$ of DLP (TC1617x image) vs. GRACoL 2013 Ref.}

The CIE L* $a^{*} b^{*}$ values associated with the CMYK+RGB colors printed image with DLP vs. G7 ColorSpace-GRACoL 2013 [CGATS21-2-CRPC6 (reference)] are compiled in Table 3. Numerical color differences $(\triangle E)$ were found when comparing the colors of the DLP printed image vs. G7 ColorSpace at all seven colors (CMYK+RGB). Also, noticeable visual color differences were found in the solid color area [lightness, color hue and chroma]. Overall, both groups of images have similar colors (see Figures $6,7,8$ and 9) with the exception of the printed image consisting of higher $L^{*}$ for green. This results in producing the higher $\Delta \mathrm{E}$ for these colors. This higher color deviation (green and cyan) could be the result of the applied DLP. Green is the darker color which produced lower $L^{*}$ value and in turn affected the higher deviation.

The 2D color gamut comparison (see Figures 6, 7, 8 and 9) reveals that the colors of the printed image closely match the reference colors. The goal was to determine the deviations among various attributes of color between these two groups of colors. The comparison is an indication that, in a color managed workflow (CMW), G7 master compliance for color matching of a target image can be achieved. Subjective judgment was not used for the color comparison. In addition to the colorimetric comparison of individual colors (Tables 3 and 4) of both groups with G7 ColorSpace, the G7 master compliance

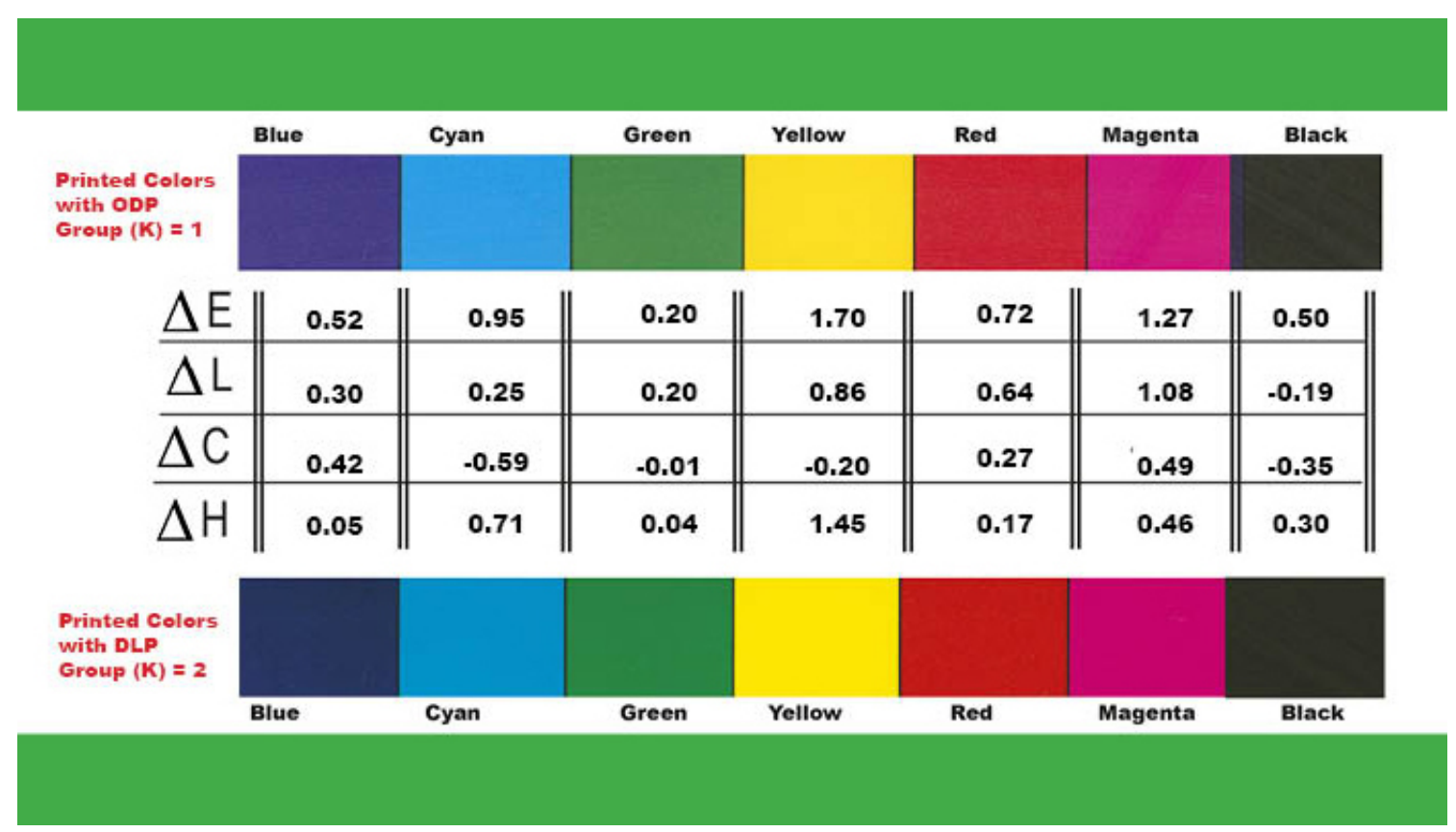

» Figure 7: Color Variations $\left(\triangle L, \triangle C^{*}, \triangle H\right.$, and $\triangle E$ ) of ODP vs. DLP [Colors presented in this chart do not represent actual $K_{1}$ vs. $K_{2}$ colors] 
colorimetric deviation ( $w \Delta C h$ and $w \Delta L$ ) values for all the three levels (G7 Grayscale, G7 Targeted and G7 Colorspace) are in close match with the established tolerances for the G7 (see Table 4, 4A, and 4B). This includes the Neutral Print Density Curve [NPDC (CMY)] and NPDC (K).

\section{Four Deviations $\left(\Delta \mathrm{L}, \Delta \mathrm{E}, \Delta \mathrm{C}^{*} \& \Delta \mathrm{H}^{*}\right)$ of OPD vs DLP}

Chroma and Hue Variation $\left(\triangle C^{*} \& \Delta H^{*}\right.$ ) values of the colors printed with ODP vs. color printed with DLP are presented in figure 8 . Interpreting $L^{*} c^{*} * h$ color space data is similar to $L^{*} a^{*} b^{*}$, but the data describes col- or differently using cylindrical coordinates instead of rectangular coordinates (see Figures 6, 7, 8 and 9). In this color space, $L^{*}$ indicates lightness, $C^{*}$ represents chroma, and $\mathrm{h}^{*}$ is the hue angle of the color. Deltas (deviations) for lightness $\left(\Delta \mathrm{L}^{*}\right)$, chroma $\left(\Delta \mathrm{C}^{*}\right)$, and hue $\left(\Delta \mathrm{H}^{*}\right)$ may be positive $(+)$ or negative $(-)$. When compared, the printed colors of ODP vs. DLP are very similar to each other (see Figures 6, 7, 8 and 9).

The $+\Delta L^{*}$ values indicate that the remaining colors are lighter. The $-\Delta C^{*}$ of cyan, green, and yellow colors indicate that they are duller in comparison with the other of the same colors in the reproduction.

\section{Table 3}

Overall Color Variation of CMYK+RGB: Printed Image (TC1617x) vs. G7 ColorSpace

\begin{tabular}{|c|c|c|c|c|c|c|c|}
\hline \multirow{3}{*}{ Color(s) } & \multicolumn{3}{|c|}{ DLP Printed Image } & \multicolumn{3}{|c|}{ G7 ColorSpace / Target } & \multirow{3}{*}{$\begin{array}{c}\text { Color } \\
\text { Difference } \\
\Delta \mathrm{E}\end{array}$} \\
\hline & $L^{*}$ & $a^{*}$ & $b^{*}$ & $L^{*}$ & a* & $b^{*}$ & \\
\hline & \multicolumn{3}{|c|}{$\begin{array}{l}\text { Color } 1 \\
\mathbf{N}=\mathbf{8 0}\end{array}$} & \multicolumn{3}{|c|}{$\begin{array}{c}\text { Color } 2 \\
\mathbf{N}=\mathbf{N} / \mathbf{A}\end{array}$} & \\
\hline White (W) & 97.06 & 2.79 & -9.33 & 97.06 & 2.79 & -9.33 & 0.00 \\
\hline Cyan & 56.75 & -30.84 & -55.13 & 57.29 & -36.82 & -55.65 & 2.28 \\
\hline Magenta & 50.11 & 75.45 & -5.72 & 49.12 & 77.81 & -7.02 & 1.20 \\
\hline Yellow & 90.00 & -3.62 & 91.56 & 90.95 & -2.43 & 92.82 & 0.91 \\
\hline Black (K) & 13.26 & 0.78 & -0.27 & 16.28 & 0.24 & -0.72 & 2.18 \\
\hline Red & 49.86 & 68.22 & 46.96 & 48.10 & 70.63 & 46.21 & 1.84 \\
\hline Green & 53.36 & -66.51 & 26.76 & 51.17 & -66.76 & 24.49 & 2.36 \\
\hline Blue & 26.76 & 19.64 & -50.73 & 25.58 & 21.14 & -50.08 & 1.54 \\
\hline TAC 300 & 24.31 & 0.42 & -2.52 & 23.52 & 0.45 & -1.31 & 1.26 \\
\hline TAC 400 & 9.84 & 0.93 & -0.36 & 8.99 & 0.17 & 0.60 & 1.56 \\
\hline
\end{tabular}

\section{Table 4}

Master Compliance Levels G7 Grayscale of OPD, DLP vs. G7

\begin{tabular}{|c|c|c|c|c|}
\hline \multirow{2}{*}{ All Metrics } & \multirow{2}{*}{$\begin{array}{c}\text { Black (K) } \\
\mathrm{w} \Delta \mathrm{L}^{*}\end{array}$} & \multicolumn{2}{|c|}{ CMY (Overlap) } & \multirow{2}{*}{ G7 Tolerance } \\
\hline & & $w \Delta L^{*}$ & w $\Delta \mathrm{Ch}$ & \\
\hline \multicolumn{5}{|c|}{ G7 Grayscale of ODP/Printed Image (Tonality/Gray Balance) } \\
\hline Average & 0.97 & 0.31 & 0.81 & 1.50 \\
\hline Maximum & 2.34 & 0.92 & 1.90 & 3.00 \\
\hline \multicolumn{5}{|c|}{ G7 Grayscale of DLP/Printed Image (Tonality/Gray Balance) } \\
\hline Average & 0.88 & 0.32 & 1.06 & 1.50 \\
\hline Maximum & 2.79 & 0.92 & 2.7 & 3.00 \\
\hline
\end{tabular}

\section{Table 4A:}

G7 Master Compliance Levels G7 Targeted of ODP, DLP vs. G7

\begin{tabular}{|c|c|c|c|c|}
\hline All Metrics & $\Delta E 2000$ & G7 Tolerance & Maximum & G7 Tolerance \\
\hline \multicolumn{5}{|c|}{ G7 Targeted of ODP/Printed Image } \\
\hline Substrate & 0.00 & 3.00 & & \\
\hline $\mathbf{K}$ & 2.29 & 5.00 & & \\
\hline CMY & & & 2.13 & 3.5 \\
\hline RGB & & & 2.45 & 4.3 \\
\hline \multicolumn{5}{|c|}{ G7 Targeted of DLP/Printed Image } \\
\hline Substrate & 0.00 & 3.00 & & \\
\hline $\mathbf{K}$ & 2.18 & 5.00 & & \\
\hline CMY & & & 2.28 & 3.5 \\
\hline RGB & & & 2.36 & 4.3 \\
\hline
\end{tabular}

\section{Table 4B:}

G7 Master Compliance Levels G7 Colorspace of ODP, DLP vs. G7

\begin{tabular}{|c|c|c|}
\hline All Metrics & $\Delta E 2000$ & G7 Tolerance \\
\hline \multicolumn{3}{|c|}{ G7 Colorspace of ODP/Printed Image } \\
\hline Average & 1.18 & 3.5 \\
\hline $95 \%$ & 2.21 & 5.0 \\
\hline \multicolumn{3}{|c|}{ G7 Colorspace of DLP/Printed Image } \\
\hline Average & 1.12 & 3.5 \\
\hline $95 \%$ & 2.15 & 5.0 \\
\hline
\end{tabular}




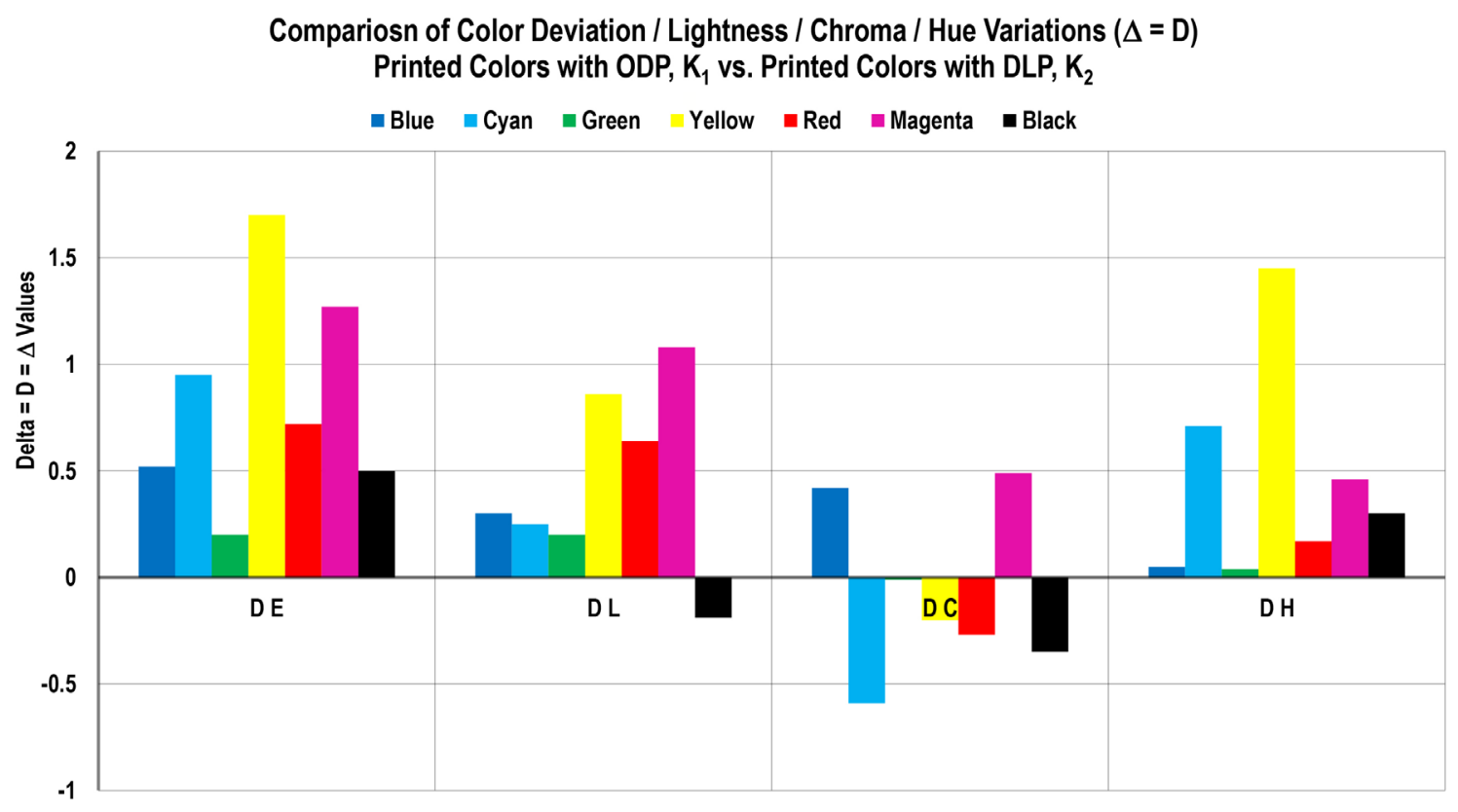

» Figure 8: Visual Color Variations $\left(\triangle L, \triangle C^{*}, \triangle H\right.$, and $\left.\triangle E\right)$ of ODP vs. DLP [Colors presented in this chart do not represent actual $K_{1}$ vs. $K_{2}$ colors]

The $+\Delta C^{*}$ of blue, red and magenta colors are brighter. The positive $\Delta H^{*}$ values of all the colors of ODP vs DLP indicate that these colors fall in counterclockwise (or clockwise) to one another, meaning these colors are almost identical visually.

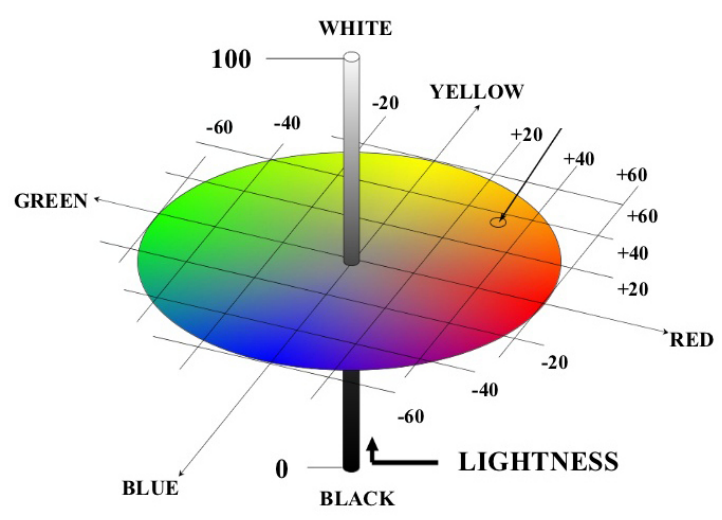

» Figure 9: CIE 2D / (3D) L* $c^{*} h *$ Color Coordinates (Courtesy of Google Images)

\section{Summary/conclusions}

This experiment used an output device ICC profile to achieve the compliance. $\mathrm{G} 7$ master compliance includes three compliance levels in the G7 master qualification: G7 Grayscale, G7 Targeted, and G7 Colorspace. These levels demonstrate G7 master capabilities of a print facility. The experiment was conducted in a Color Managed Digital Printing Workflow (CMDPW). It was aimed at achieving the $\mathrm{G} 7$ master compliance through an ICC based color managed workflow (CMW) by applying the ODP and DLP as two independent groups.
The G7 calibration method, using the P2P251x target, was NOT used to derive the device NPDC to compare with G7 NPDC for print (or press) runs 1, 2, 3, etc.

The conclusions of this study are based upon an analysis of colorimetric data, visual assessment, and associated findings. The experiment analyzed the comparison of G7 compliance of two print profiles (ODP and DLP). The guiding objectives of this study allowed testing of an accepted color management practice to gain a better understanding of the presumptions associated with the application of an output device profile (ODP) and device link profile (DLP). The experiment examined the importance of calibration, characterization and the color evaluation processes of the digital press which was capable of printing colors to match or be in proximity of $\mathrm{G} 7$ master compliance levels.

It is evident that integration of device profiles (ODP or DLP) is important in a CMW and it also enables/allows the workflow process to meet the G7 compliance levels via an ICC based CMW, instead of using G7 calibration methodology. Selection of output profile (ODP or DLP) is based on individual preference because printing with both the profiles proved to be within the G7 compliance (G7 Grayscale, G7 Targeted, and G7 Colorspace). Use of DLP reduces the number of steps to follow in the workflow at the RIP of the digital press for managing the color printing because most of the print parameters (calibration, destination profile, source profile, screening option, etc.) are already embedded in the DLP. This study represented specific printing or testing conditions. The images, printer, instrument, software, and paper that were utilized are important factors to consider when evaluating the results. The findings of the study cannot be 
generalized to other digital printing workflow. However, other graphic arts educators, industry professionals, and researchers may find this study meaningful and useful.

\section{References}

Avramovic, D. \& Novakovic, D. (2012) Influence of Printing Surface Attributes on Print Quality in Electrophotography. Technical Gazette. 19 (2), 295-301.

Chromix, Inc. (2019) Curve 4.2.4 Software Manual. Seattle, Chromix, Inc.

Committee for Graphic Arts Technologies StandardsCGATS (2003) Graphic technology - spectral measurement and colorimetric computation for graphic arts image. (ANSI/CGATS.5-2003). Reston, The Association for Suppliers of Printing and Publishing Technologies.

Glass, G. V. \& Hopkins, K. D. (1996) Statistical Methods in Education \& Psychology. Boston, Allyn \& Bacon.

Goodhard, M. M. \& Wilhelm, H. (2003) A new test method based on CIELAB colorimetry for evaluating the permanence of pictorial images. Wilhelm Imaging Research, Inc. Available from: http://www.wilhelm-research.com [Accessed: 20th August 2019].

International Digital Enterprise Alliance- IDEAlliance (2014) IDEAlliance Guide to Print Production. Alexandria, IDEAlliance.

Morovic, J., Green, P. \& MacDonald, L. (2002) Color Engineering. New York, John Wiley \& Sons. pp. 297-314.

Wales, T. (2009) Paper: The Fifth Color. Alexandria, IPA Bulletin- International Prepress Association (IPA)- Now part of IDEAlliance. Available from: http://idealliance. org/files/2008_03tech.pdf [Accessed: 20th August 2019].

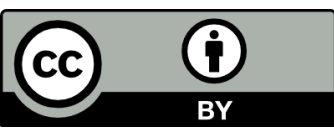

(C) 2021 Authors. Published by the University of Novi Sad, Faculty of Technical Sciences, Department of Graphic Engineering and Design. This article is an open access article distributed under the terms and conditions of the Creative Commons Attribution license 3.0 Serbia (http://creativecommons.org/licenses/by/3.0/rs/). 